\title{
Alleviation of Neurotoxicity by Microglial Human Siglec-11
}

\author{
Yiner Wang and Harald Neumann \\ Neural Regeneration Group, Institute of Reconstructive Neurobiology, University of Bonn, 53127 Bonn, Germany
}

Sialic acid-binding Ig superfamily lectins (Siglecs) are members of the Ig superfamily that recognize sialic acid residues of glycoproteins. Siglec-11 is a recently identified human-specific CD33-related Siglec that binds to $\alpha 2,8$-linked polysialic acids and is expressed on microglia, the brain resident innate immune cells. Polysialylated neuronal cell adhesion molecule (PSA-NCAM) is a putative ligand of Siglec-11. We observed gene transcription and protein expression of Siglec-11 splice variant 2 in human brain tissue samples by RT-PCR and Western blot analysis. Siglec-11 was detected on microglia in human brain tissue by immunohistochemistry. Human Siglec-11 splice variant 2 was ectopically expressed by a lentiviral vector system in cultured murine microglial cells. Stimulation of Siglec-11 by crosslinking suppressed the lipopolysaccharides (LPS)-induced gene transcription of the proinflammatory mediators interleukin-1 $\beta$ and nitric oxide synthase- 2 in microglia. Furthermore, phagocytosis of apoptotic neuronal material was reduced in Siglec-11 transduced microglia. Expression of PSA-NCAM was detected on microglia and neurons by immunohistochemistry and RT-PCR. Coculture of microglia transduced with Siglec-11 and neurons demonstrated neuroprotective function of Siglec-11. The neuroprotective effect of Siglec-11 was dependent on polysialic acid (PSA) residues on neurons, but independent on PSA on microglia. Thus, data demonstrate that human Siglec-11 ectopically expressed on murine microglia interacts with PSA on neurons, reduces LPS-induced gene transcription of proinflammatory mediators, impairs phagocytosis and alleviates microglial neurotoxicity.

\section{Introduction}

Sialic acid-binding Ig superfamily lectins (Siglecs) are type 1 membrane proteins displaying an N-terminal Ig-like variable (Vset Ig-like) domain that binds sialic acid and a variable number of Ig-like constant region type 2 (C2-set Ig-like) domains (Crocker, 2005; Varki and Angata, 2006; Crocker et al., 2007; Varki, 2009). Siglecs can be divided into two subsets on the basis of their sequence similarity and evolutionary conservation (Varki and Angata, 2006). One subset consists of CD33-related Siglecs, which show a similarity of $\sim 50-99 \%$ in their protein sequences. A striking feature of the CD33-related Siglecs is that they were evolving very rapidly. In humans, there are 10 CD33-related Siglecs and 1 Siglec-like protein, whereas there are only 5 CD33related Siglecs in mice, which seem to have undergone wholesale loss of CD33-related Siglec genes (Angata et al., 2006, 2007; Crocker et al., 2007; Cao et al., 2008, 2009; Varki, 2009). Although CD33-related Siglecs have unique expression profiles, they are predominantly found on leukocytes. Furthermore, accumulating data indicate that they are important regulators of the innate immunity (Crocker, 2005; Crocker et al., 2007; Cao et al., 2009; Carlin et al., 2009; Varki, 2009).

\footnotetext{
Received Aug. 12, 2009; revised Dec. 15, 2009; accepted Dec. 24, 2009.

This project was supported by the DANA Foundation, Deutsche Forschungsgemeinschaft (KF0177, SFB704), and the European Union (LSHM-CT-2005-018637). The Neural Regeneration Group at the University Bonn LIFE \& BRAIN Center is supported by the Hertie-Foundation and Walter-and-Ilse-Rose-Foundation. We thank Dr. Clussmann and Dr. Dietrich for support in obtaining human brain tissue samples. We thank Dr. A. Varki for helpful discussions. We thank Jessica Schumacher and Rita Hass for excellent technical support of cultures and molecular biology.

The authors declare no competing financial interests.

Correspondence should be addressed to Harald Neumann, Neural Regeneration Unit, Institute of Reconstructive Neurobiology, University Bonn, Sigmund-Freud-Straße25, 53127 Bonn, Germany. E-mail: hneuman1@ uni-bonn.de.

DOI:10.1523/JNEUROSCI.3940-09.2010

Copyright $\odot 2010$ the authors $\quad 0270-6474 / 10 / 303482-07 \$ 15.00 / 0$
}

The recently discovered Siglec-11 belongs to the CD33-related subfamily of human Siglecs (Angata et al., 2002). The protein deduced from the full-length cDNA of Siglec-11 consists of five extracellular Ig-like domains, a single-pass transmembrane domain, and a cytosolic tail (Angata et al., 2002). Like most other members of the CD33-related Siglecs, it has several immunoreceptor tyrosine-based inhibitory motifs (ITIMs) in the cytoplasmic domain, which interact with the Src homology domain 2-containing phosphatase-1 (SHP-1) and SHP-2 upon tyrosine phosphorylation (Angata et al., 2002). Interestingly, SHP-1 has been shown to be involved in anti-inflammatory signaling of microglia (Zhao et al., 2006). However, Siglec-11 also has several novel features superior to the other CD33-related Siglecs. First, it binds specifically to $\alpha 2,8$-linked sialic acids, also known as $\alpha 2,8$ linked $N$-acetylneuraminic acid (NeuAc- $\alpha 2-8$ ) (Angata et al., 2002), but the ligand molecule modified by $\alpha 2,8$-linked sialic acids and recognized by Siglec-11 has not been fully identified. Thus, polysialic acid (PSA), which prominently is attached to glycoproteins in the CNS (Rutishauser, 2008), is a putative ligand for Siglec-11. Second, expression of Siglec-11 was not found on peripheral blood leukocytes, but on various tissue macrophages including brain microglia (Angata et al., 2002). Analysis of genome databases indicated that Siglec-11 has no mouse ortholog (Hayakawa et al., 2005).

Microglial cells are resident CNS immune cells that execute innate immunity and participate in adaptive immune responses (Biber et al., 2007; Hanisch and Kettenmann, 2007; Ransohoff and Perry, 2009). In response to injury, ischemia, and inflammatory stimuli, microglial cells assume an activated phenotype associated with proliferation, migration to the site of injury, phagocytosis of cellular debris, and elaboration of both neurotoxic and neurotrophic effects (Ransohoff and Perry, 2009). In 


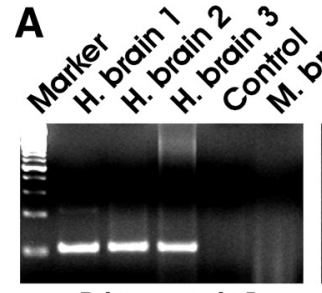

Primer pair 1

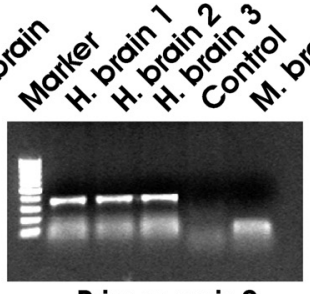

Primer pair 2

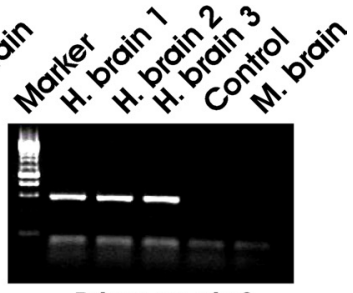

Primer pair 3
B

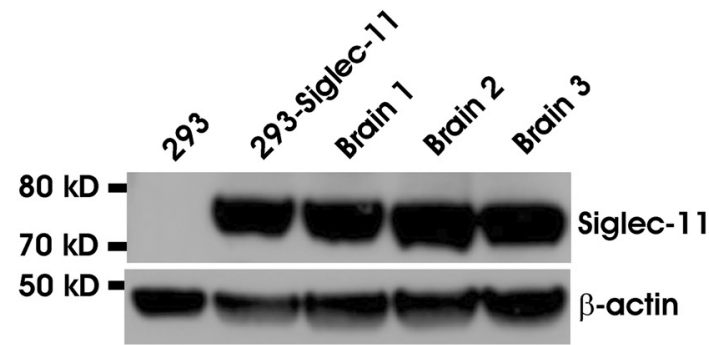

C
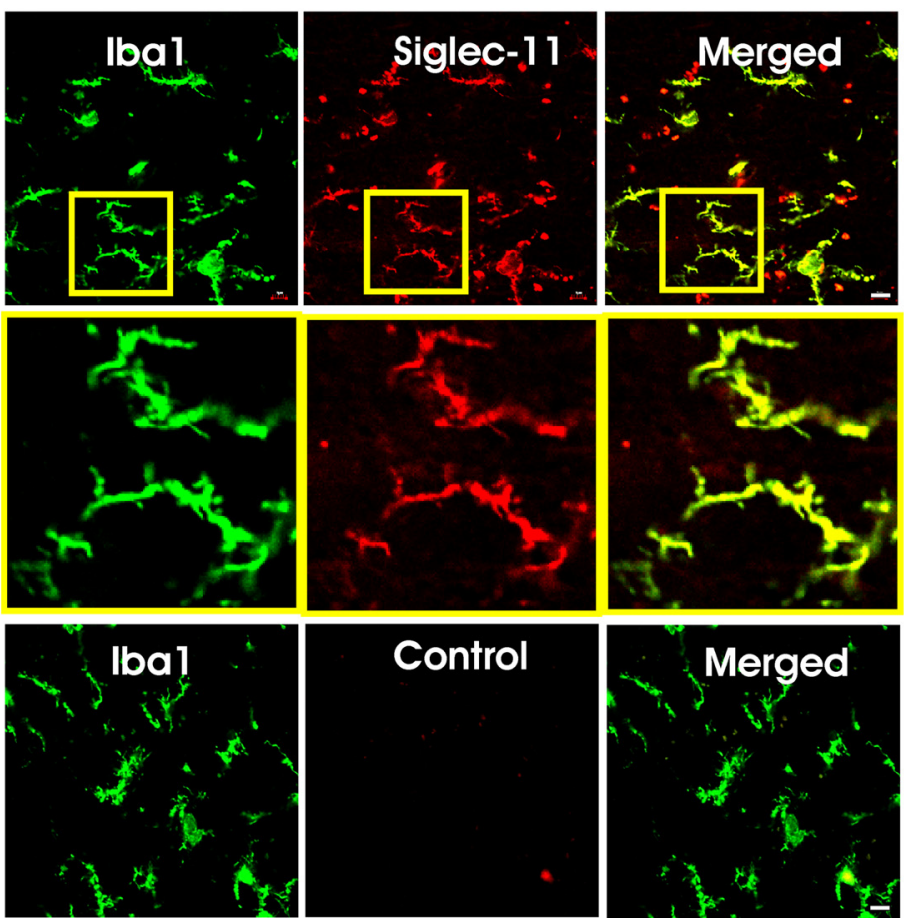

Figure 1. Detection of the splice variant 2 of Siglec-11 in human brain tissue. $A$, Gene transcript analysis of three different human brain tissues samples (H. brain 1,2, and 3) by RT-PCR. Amplification with three different pairs of primers (primer pair 1,2, and 3) from distinct exons resulted in PCR products of $103 \mathrm{bp}, 352 \mathrm{bp}$, and $294 \mathrm{bp}$, respectively. All three PCR products were sequenced and showed gene sequence of Siglec-11 splice variant 2. Control, RNA of tissue sample 1 without reverse transcription. $M$. brain, CDNA from mouse brain tissue. Marker, 100 bp ladder. $\boldsymbol{B}$, Western blot analysis of protein lysates from distinct human brain tissue samples (brain 1,2, and3), normal 293 cells (293), and 293 cells transduced with the Siglec-11 vector (293-Siglec-11). Siglec-11 protein was detected in the brain tissue and the positive control (293-Siglec-11). Antibody directed against $\beta$-actin, which labeled a $42 \mathrm{kDa}$ protein, was applied as control. $C$, Immunofluorescence analysis of Siglec-11 in human brain tissue samples. Brain tissue sections were double immunolabeled with antibodies directed against Siglec-11 and Iba1. Siglec-11 expression was detected in microglial cells by confocal laser microscopy. Background staining was determined by control antibodies (control). Scale bars, $15 \mu \mathrm{m}$.

most neurodegenerative diseases, microglial cells are activated and have bipolar effector function. On the one hand, they can produce proinflammatory mediators, such as interleukin- $1 \beta$ (IL$1 \beta$ ), tumor necrosis factor- $\alpha$ (TNF- $\alpha$ ), and nitric oxide (NO), which are potent inducers of neuronal damage and cell death (Ransohoff and Perry, 2009). On the other hand, microglia can also express anti-inflammatory cytokines and initiate negative feedback pathways that result in repair, resolution of inflammation, and turning back to tissue homeostasis (Cardona et al., 2006; Neumann et al., 2009; Ransohoff and Perry, 2009). Several molecules were described to downregulate microglial activity. An inhibitory function of CD200, which is expressed on neurons and acts on CD200 receptors of microglia, was observed in the animal model experimental autoimmune encephalomyelitis after facial nerve transaction and toxoplasma encephalitis (for review, see Biber et al., 2007). Neurotoxic microglial activity was also suppressed by the neuronal chemokine CX3CL1 signaling via the microglial chemokine receptor CX3CR1 as shown in neuron-microglia cocultures (Zujovic et al., 2000) and distinct disease models analyzed in CX3CR1-deficient mice (Cardona et al., 2006).

In our study, we analyzed the role of Siglec-11 in the interaction between microglia and neurons. Our findings assign Siglec-11 as an inhibitory receptor of inflammatory responses of microglia and open a new avenue for modulating microglial neurotoxicity.

\section{Materials and Methods}

Experimental animals and human tissue samples. All animal experiments and studies on human tissue samples have been approved by the authors' institutional review boards and by the local government and have been conducted according to the principles expressed in the Helsinki Declaration.

Gene transcript and protein expression analysis in human brain tissue. RT-PCR analysis, Western blot analysis, and immunohistochemistry were performed to detect gene transcription and protein expression of Siglec-11 in brain tissue samples from in total 6 patients $(3$ female, 3 male, age ranging from 8 to 43 years) consisting of amygdala or/and hippocampus, excluding brain tumor (details are presented in the supplemental Material and Methods, available at www.jneurosci. org as supplemental material).

Primary microglial and neuronal culture and immunocytochemistry. Primary microglia and neuronal cells were prepared as described previously (Takahashi et al., 2005; Gorlovoy et al., 2009). Cells were either untreated or treated with $0.5 \mathrm{U} / \mathrm{ml}$ endo- $N$-acetylneuraminidase (EndoN) for $5 \mathrm{~h}$ to remove PSA. Cells were fixed with $4 \%$ PFA (Sigma-Aldrich) and incubated sequentially with the primary mouse monoclonal antibody directed against $\beta$-tubulin-III (1:500, Sigma-Aldrich) or Ibal-specific rabbit antibody (1:2000, Wako), secondary cy3-conjugated goat antibody directed against mouse or rabbit Ig (1:200, Jackson ImmunoResearch Laboratories), mouse monoclonal antibody directed to polysialylated neuronal cell adhesion molecule (PSA-NCAM) (1:500, Millipore), and goat antibody directed against mouse Ig conjugated to FITC (1:200, Jackson ImmunoResearch Laboratories).

Lentiviral vector system. The cDNA clone of Siglec- 11 variant 2 was obtained from the German genetic material source center. The following 
vectors were cloned into the lentiviral backbone PLL3.7 after the U6 promoter was removed and the sequence was confirmed by automated sequencing: PromoterCmv-3xflagSiglec-11-GFP (flag-Siglec-11 vector), PromoterCmv-Siglec-11 (Siglec-11 vector), and PromoterCmv-GFP (GFP control vector). Lentiviral transduction was performed as described by the manufacturer's instructions (Virapower; Invitrogen) and as described previously (Takahashi et al., 2005) (details are presented in the supplemental Material and Methods, available at www.jneurosci.org as supplemental material).

Phagocytosis assay of apoptotic neuronal material. Murine primary microglia were lentivirally transduced with Siglec-11 or GFP control vector and challenged with red fluorescent labeled apoptotic neuronal material. Uptake of apoptotic material was analyzed by confocal fluorescence microscopy (details are presented in supplemental Material and Methods, available at www.jneurosci.org as supplemental material).

Real-time RT-PCR analysis of gene transcription of proinflammatory mediators. Microglia cells were isolated from mixed glial culture, transduced with flag-Siglec-11 or GFP control vector and cultured for $48 \mathrm{~h}$ to achieve effective expression of targeted genes. Cells were then cultured on dishes coated with flag-specific antibodies $(10 \mu \mathrm{g} / \mathrm{ml}$, Sigma-Aldrich $)$ or isotype control antibody ( $10 \mu \mathrm{g} / \mathrm{ml}$, Sigma-Aldrich) and treated with 500 $\mathrm{ng} / \mathrm{ml}$ lipopolysaccharides (LPS) (Sigma-Aldrich) for $48 \mathrm{~h}$. RNA was isolated from microglia by the RNeasy Mini Kit (Qiagen). Reverse transcription and semiquantitative RT-PCR were performed with specific oligonucleotides and SYBR Green PCR Master Mix (Applied Biosystems) using the ABI 5700 Sequence Detection System (PerkinElmer) and amplification protocol for the ABI 5700 Sequence Detection System. Quantification using the delta-CT method was carried out.

Microglia-neuron coculture and immunocytochemistry of cocultured cells. Primary microglia were transduced with Siglec-11 or GFP control vector and cocultured with neurons. Neurite and neuronal cell body density were quantified (details are presented in supplemental Material and Methods, available at www.jneurosci.org as supplemental material).

\section{Results}

Detection of Siglec-11 splice variant 2 gene transcripts in human brain tissue

Human brain tissue obtained from patients who underwent epilepsy surgery were analyzed by RT-PCR using primers designed to specifically detect the distinct known splice variants of Siglec-11 (supplemental Fig. S1, available at www.jneurosci.org as supplemental material). Data demonstrated that splice variant 2 of Siglec-11 was transcribed in human brain tissue. The splice variant 2 is 288 bp shorter than the full-length gene transcript and lacks one exon, which is coding the Ig-like domain 5 in the extracellular part. This shorter splice variant was consistently detected in all analyzed brain tissue samples obtained from distinct patients (Fig. 1A). Direct sequencing of the amplified PCR products confirmed that exactly the exon coding the fifth Ig-like domain was missing.

\section{Detection of Siglec-11 protein in human brain tissue}

Western blot and immunohistochemistry analysis were performed to analyze the protein expression of Siglec-11 in human brain tissue samples. As a positive control Siglec- 11 splice variant 2 derived from a human cDNA clone was placed into a lentiviral backbone, confirmed by automated sequencing and transduced into 293 cells (supplemental Fig. S1, available at www.jneurosci. org as supplemental material). The expression of Siglec-11 on the cell surface of 293 cells was confirmed by flow cytometry analysis (supplemental Fig. S1, available at www.jneurosci.org as supplemental material). Siglec-11 was detected in the lysates of the human brain samples by Western blot analysis as a protein of $\sim 80$ $\mathrm{kDa}$ (Fig. $1 B$ ). The Siglec-11 band in the blot showed the same size as the Siglec-11 from protein lysate of the 293 cells expressing the Siglec-11 splice variant 2 (Fig. $1 B$ ). Furthermore frozen hu-
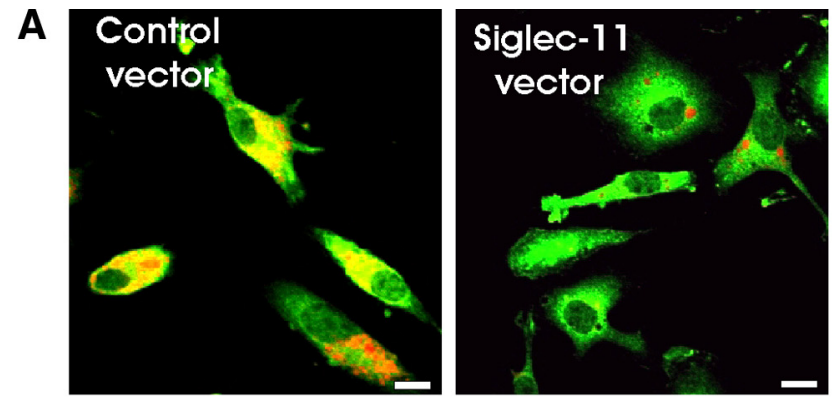

B

IL-1 $\beta$
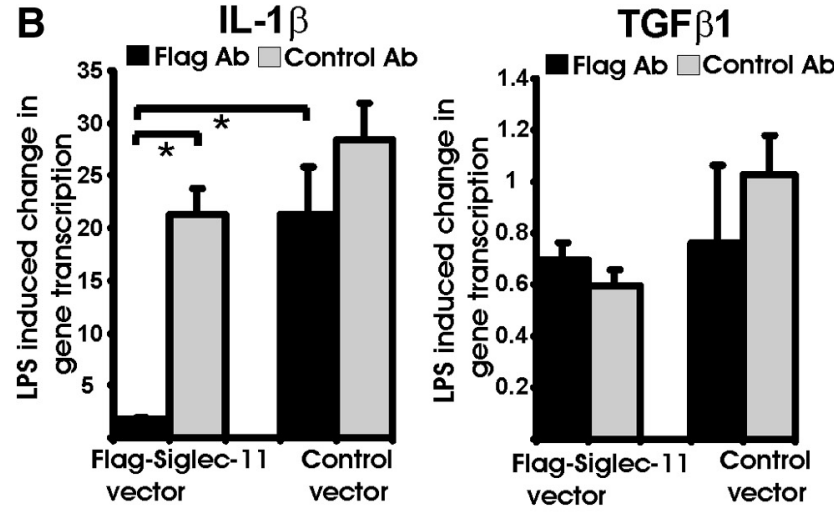

TNF $\alpha$
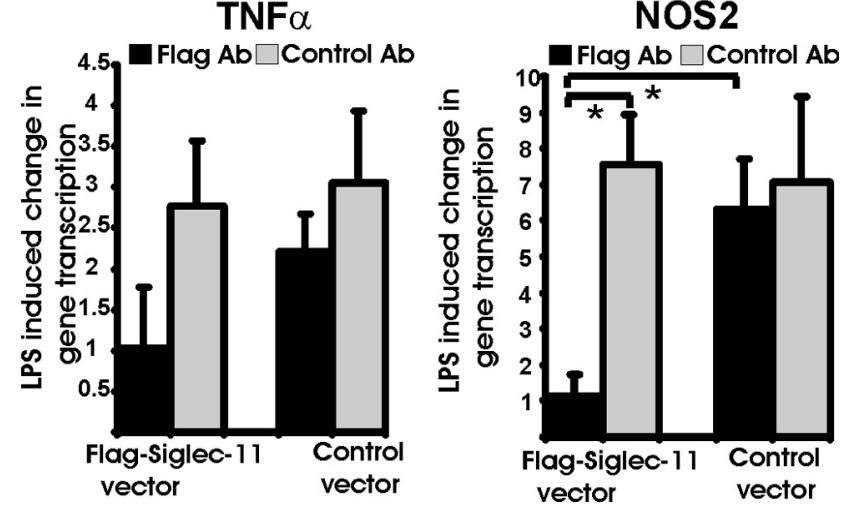

Figure 2. Reduced phagocytosis of apoptotic neuronal material and suppression of proinflammatory gene transcription by Siglec- 11 transduction in murine microglia. $\boldsymbol{A}$, Representative images showing murine microglia transduced with the control (left image) or Siglec-11 vector (right image) and stained with antibodies directed against lba1 (labeled with green fluorescence dye). Scale bars, $10 \mu \mathrm{m}$. $\boldsymbol{B}$, Suppression of proinflammatory gene transcription by Siglec-11 transduction in murine microglia. Primary brain-derived microglial cells transduced with a flag-tagged Siglec-11 (flag-Siglec-11) vector or the GFP control vector (Control vector) were cultured on plates precoated with antibodies directed against the flag-tag (Flag Ab) or the isotype control antibody (Control Ab). Microglial cells were either unstimulated or stimulated with $500 \mathrm{ng} / \mathrm{ml}$ LPS. Gene transcripts of microglial cells were studied by real-time RT-PCR. Cross-linking of Siglec-11 prevented the LPS-induced increase in IL-1 $\beta$ and NOS2 gene transcription in Siglec-11, but not in control vector transduced microglia. Data are shown as mean \pm SEM and as relative increase in LPS-induced gene transcription; number of independent experiments $n=4$. ${ }^{*} p<0.05$, ANOVA followed by Bonferroni multiple-comparison test.

man brain sections were double immunostained with antibodies directed against Siglec-11 and the typical microglial marker ionized calcium binding adaptor molecule 1 (Iba1). Siglec-11 colocalized with Ibal stained cells, demonstrating that Siglec-11 was expressed on brain microglia (Fig. 1C).

Reduced phagocytosis of apoptotic neuronal material by Siglec-11-expressing murine microglia

Apoptotic neuronal material was prepared by treating neurons derived from C57BL/6 mouse embryonic hippocampal brain tis- 

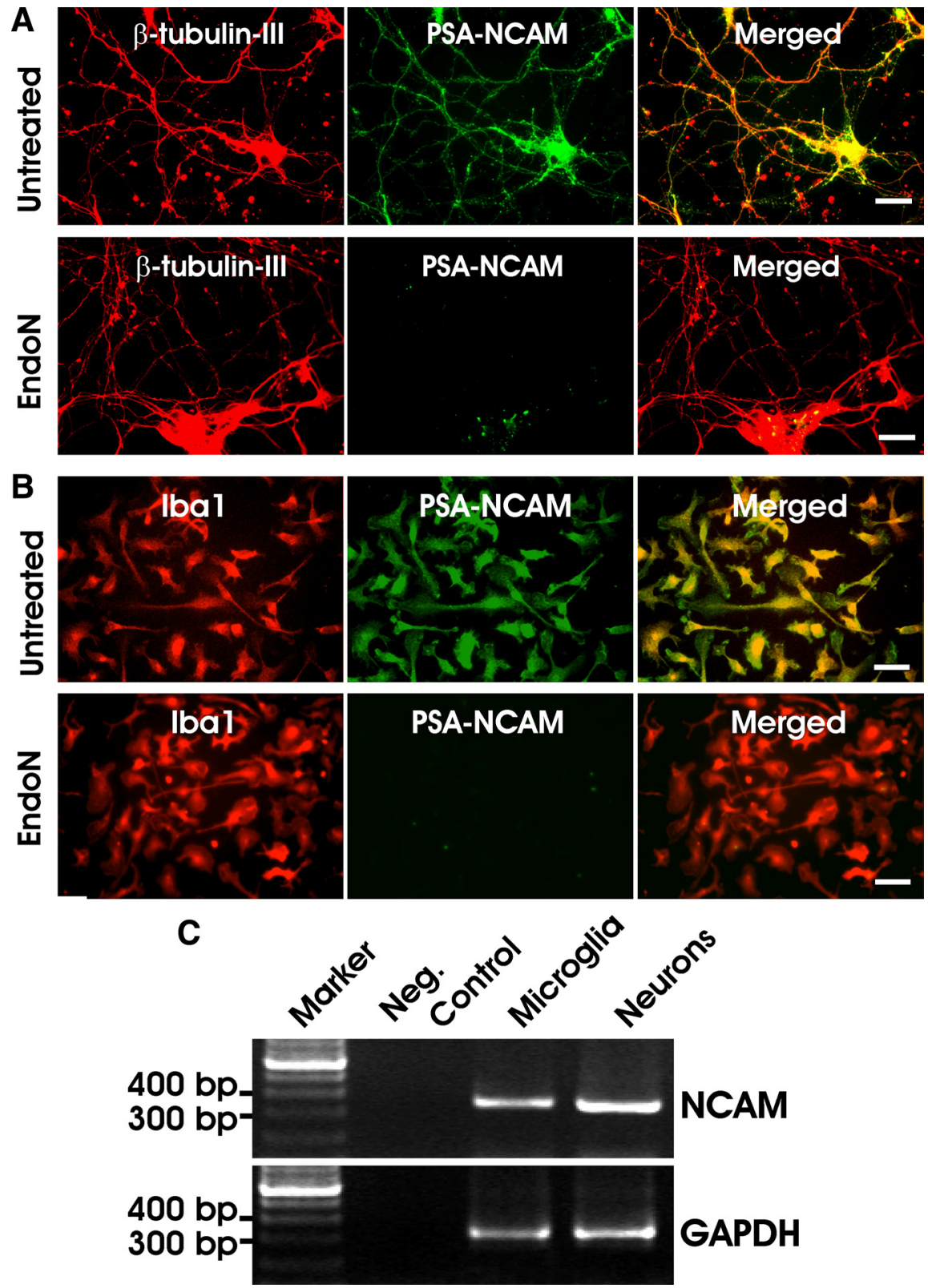

Figure 3. Detection of PSA-NCAM expression on cultured neurons and microglia. $A$, Hippocampal neurons were cultured from embryonic brain tissue. Neurons were double immunostained with specific antibody recognizing the PSA-containing form of NCAM and $\beta$-tubulin-III-specific antibody. Neurons were either untreated or treated with EndoN for $5 \mathrm{~h}$ before fixation. PSANCAM was detected on untreated neuronal cell bodies and neurites, but was absent on EndoN-treated neurons. Scale bar, $50 \mu \mathrm{m} . \boldsymbol{B}$, Microglial cells were obtained from postnatal mixed glial cultures. Microglia were immunostained with monoclonal anti-PSA-NCAM antibody. Microglial cells were identified by double labeling with antibodies directed against lba1. No immunostaining of PSA-NCAM was detected after removal of polysialic acids by EndoN. Scale bar, $50 \mu \mathrm{m}$. C, RT-PCR was performed using microglial and neuronal CDNAs. NCAM gene transcripts were detected in both microglia and neurons. Amplification of GAPDH was applied to confirm the integrity of the materials. Neg. control, Microglial mRNA samples without reverse transcription.

sue with okadaic acid. Apoptotic cellular material was labeled with CellTracker CM-DiI membrane dye. Capacity of murine microglia transduced with the Siglec-11 vector to phagocytose apoptotic neural material was compared to that of microglia transduced with the control vector (Fig. 2A). Microglia expressing Siglec-11 showed reduced phagocytosis capacity. In detail, $71.65 \pm 14.32 \%$ of control vector transduced microglia phagocytosed apoptotic neuronal material within $1 \mathrm{~h}$, while only $30.70 \pm 14.37 \%$ of Siglec-11 vector transduced microglia showed phagocytosis of apoptotic neuronal material ( $n=4 ; p \leq 0.01$, two-tailed $t$ test) (Fig. $2 A)$.

Downregulation of inflammatory mediators after stimulation of human Siglec-11 transduced in murine microglia

Murine primary microglial cells were transduced with a lentiviral vector to overexpress flag tagged human Siglec-11 variant 2 (flagSiglec-11) to enable stimulation of Siglec-11 by cross-linking with flag-specific antibodies. Microglial cells expressing flag-Siglec-11 were cultured on plates precoated with a monoclonal antibody directed against the flag epitope or an isotype control antibody. Cells were either unstimulated or stimulated with $500 \mathrm{ng} / \mathrm{ml}$ LPS. Gene transcripts of microglial cells were studied after $48 \mathrm{~h}$ of culture by real-time RT-PCR. Data showed that after cross-linking of flag-Siglec-11 by the flag-specific antibodies, the relative LPS-induced gene transcription level of interleukin-1 $\beta$ (IL-1 $\beta$ ) was reduced in the Siglec-11 vector transduced cells (1.81 \pm 0.11 , mean \pm SEM) compared to the control vector transduced cells $(21.37 \pm 4.44)$ (Fig. $2 B$ ). The LPS-induced gene transcription of nitric oxide synthase-2 (NOS2) also was lower in flag-Siglec-11-expressing cells $(1.21 \pm 0.59)$ than in the control vector transduced cells $(6.32 \pm 1.39)$ (Fig. 2B). Although there was no significant difference in the relative level of transforming growth factor- $\beta 1$ and tumor necrosis factor- $\alpha$ (TNF- $\alpha$ ), the LPS-induced gene transcription of TNF- $\alpha$ was also slightly suppressed after cross-linking of flag-Siglec-11 (Fig. $2 B)$. Thus, data demonstrated that crosslinking of flag-Siglec-11 decreased gene transcription of LPS-induced proinflammatory mediators in microglia.

\section{Expression of PSA-NCAM on neurons and microglia}

Neurons from C57BL/6 mouse embryonic hippocampal brain tissue were cultured, then fixed and immunostained with antibodies directed against PSANCAM and $\beta$-tubulin-III. Expression of PSA-NCAM was detected on neuronal cell bodies and neurites as identified by double labeling with antibodies directed against $\beta$-tubulin-III (Fig. 3A). After treatment of neurons with EndoN, PSA was enzymatically removed and PSA-NCAM was undetectable (Fig. 3A). Microglial cells identified by immunolabeling with antibody directed against Ibal also showed coimmunostaining for PSA-NCAM, which was undetectable after treatment with EndoN (Fig. 4B). The expression of NCAM was confirmed by RT-PCR using primers derived from sequences present in all NCAM isoforms (BallyCuif et al., 1993). A 337 bp NCAM PCR product was detected from microglial cDNA as well as neuronal cDNA (Fig. 3C). 

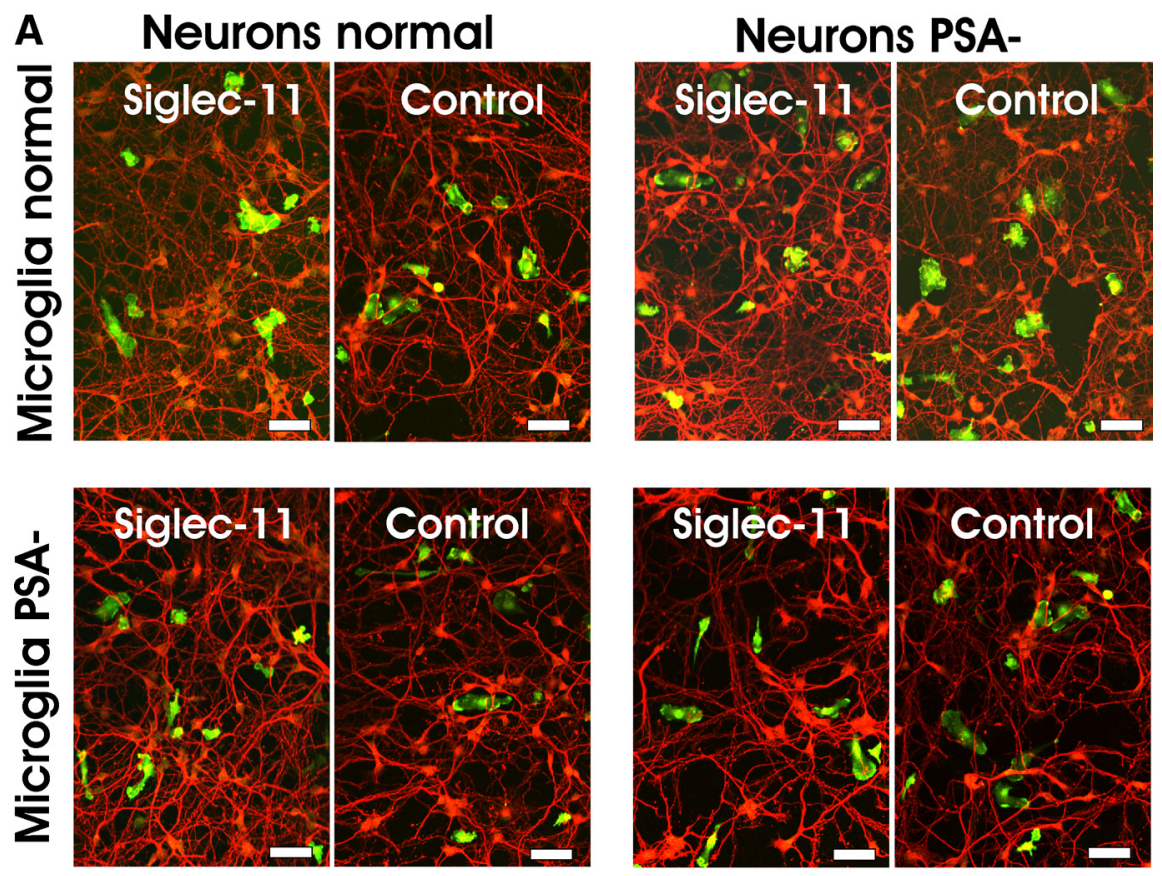

B

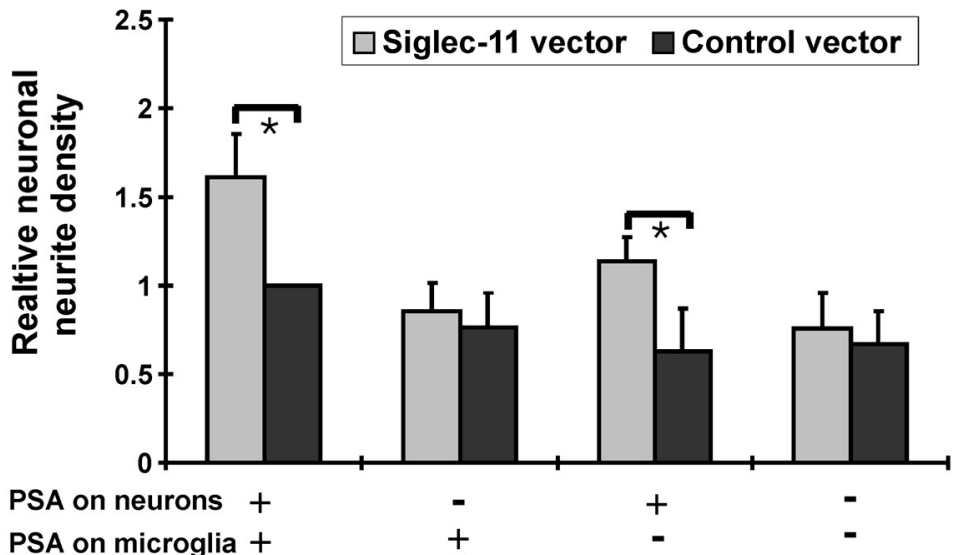

C

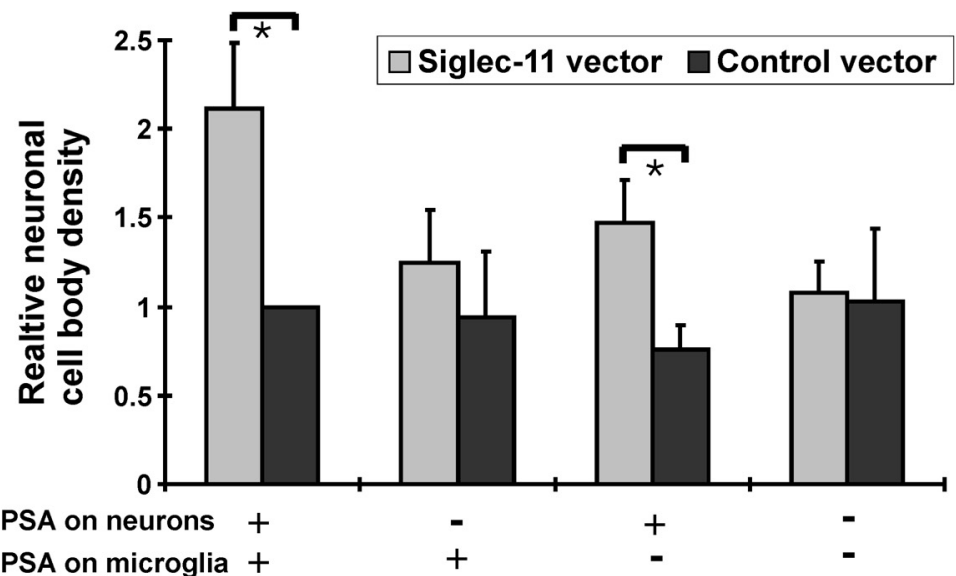

Figure 4. Neuroprotection of microglial Siglec-11 in microglia-neuron cocultures dependent on PSA on neurons. $\boldsymbol{A}$, Microglial cells were lentivirally transduced with the Siglec-11 (Siglec-11) or control vector (Control). PSA was removed from neurons and/or microglia by EndoN treatment. Cells were washed and cocultured for $48 \mathrm{~h}$. Cells were fixed and double-immunostained with antibodies directed against $\beta$-tubulin-III (green signal) and lba- 1 (red signal). Confocal images were obtained by laser scanning microscopy. Neurite density was higher in cocultures with Siglec-11-expressing microglia and neurons having PSA residues on their glycoproteins. Scale bars, $50 \mu \mathrm{m}$. B , Statistics analysis of neurite density. The number of neurites was determined and values were normalized to those of cocultures with control vector transduced microglia. Data are shown as mean \pm SEM; number of independent experiments $n=5,{ }^{*} p<0.05$, ANOVA followed by Bonferroni multiple-comparison test. C, Statistics analysis of neuronal cell body survival. The number of survived neuronal cell body in each treatment was counted and values were normalized
Neuroprotective effect of Siglec-11 in neuron-microglia coculture

Microglial cells were transduced with the Siglec-11 vector and cocultured with neurons for $48 \mathrm{~h}$. Analysis of neurites immunolabeled by antibodies directed against $\beta$-tubulin-III revealed that in cocultures between PSA-containing neurons and Siglec-11 vector transduced microglia neurite density was higher than in cocultures with control vector transduced microglia (Fig. 4A). In detail, relative neurite density was $1.61 \pm 0.24$ (mean \pm SEM) for Siglec-11 vector versus 1 for control vector transduced microglia when PSA was present on both neurons and microglia, and $1.14 \pm 0.14$ versus $0.63 \pm 0.24$ when PSA was present only on neurons, but not on microglia (Fig. 4B). This effect was not observed when PSA was removed from the neurons (Fig. 4B). Then, relative neurite density was $0.85 \pm 0.16$ for Siglec-11 vector versus $0.79 \pm 0.24$ for control vector transduced microglia when PSA was still present on microglia, and $0.76 \pm 0.19$ versus $0.67 \pm 0.19$ when PSA was absent on both neurons and microglia. A similar neuroprotective effect of Siglec-11 was also observed by analysis of neuronal cell body density (Fig. 4C). When PSA was present on neurons, the cocultures of neurons with Siglec-11 vector transduced microglia resulted in higher neuronal cell body density than those of neurons cocultured with control vector transduced microglia. In detail, relative neuronal cell body density was $2.11 \pm 0.37$ for Siglec-11 vector versus 1 for control vector transduced microglia when PSA was present on both neurons and microglia, and $1.48 \pm 0.24$ versus $0.71 \pm 0.13$ when PSA was present only on neurons, but not on microglia. This protective function of Siglec-11 on neuronal cell body survival was not observed when PSA was removed from neurons (Fig. 4C). Then, the relative neuronal cell body density was $1.25 \pm 0.29$ for Siglec-11 vector versus $0.94 \pm 0.38$ for control vector transduced microglia when PSA was removed from neurons, but present on microglia, and $1.08 \pm 0.18$ versus $1.03 \pm$ 0.42 when PSA was absent on both neurons and microglia. In summary, data demonstrated that more neurites and neuronal cell bodies survived in cocul-

$\leftarrow$

to those of cocultures with control vector transduced microglia. Data are shown as mean $\pm \mathrm{SEM}$; number of independent experiments $n=5,{ }^{*} p<0.05$, ANOVA followed by Bonferroni multiple-comparison test. 
tures with Siglec-11 transduced microglia compared to control vector transduced microglia, demonstrating a neuroprotective effect of Siglec-11. The PSA residues expressed on neurons, but not those on microglia contributed to the neuroprotective function of microglial Siglec-11.

\section{Discussion}

Siglec-11 as first described by Angata et al. (2002) was characterized as a gene coding one transcript. However, sequence databases of expressed sequence tags indicated that at least one further splice variant of this gene is transcribed (dbEST, GenBank, NIH). Using Siglec-11-specific primers derived from different exons of the coding region, we identified the Siglec-11 splice variant 2 transcript in all analyzed human brain tissue samples obtained from different patients. The protein size of Siglec-11 detected by Western blotting in human brain tissue samples was identical to the Siglec-11 protein expressed in 293 cells after transduction with the gene Siglec-11 splice variant 2 . Recently, Siglec-16 was found and described as being evolved from Siglec-11 as a DAP12-associated receptor expressed in macrophages (Cao et al., 2008). The Siglec-16 protein shares a high percentage $(\sim 99 \%)$ of amino acid identity with Siglec-11 especially at the extracellular part, but its transmembrane domain contains a charged lysine residue and it has a short cytoplasmic tail without ITIM. The Siglec-16 protein has a molecular weight of $\sim 58 \mathrm{kDa}$ (Cao et al., 2008). Although our immunohistochemistry could not discriminate between Siglec-11 and Siglec-16 since the antibody was raised against the highly homologous extracellular part of Siglec-11, the RT-PCR products obtained by using Siglec-11-specific primers and the molecular weight of the Western blot analysis firmly supported the expression of Siglec-11 in human brain tissue. Thus, our data demonstrated that the shorter splice variant 2 of Siglec- 11 is expressed on microglia in human brain. However, it is also possible that the splice variants of Siglec-11 are differently transcribed in distinct tissue types. From a structural point of view, the Siglec-11 splice variant 2 should not differ from the full-length Siglec- 11 in its property to recognize a ligand, because the known functionally relevant binding sites of Siglecs are localized at the N-terminal first V-set Iglike domain, which binds sialic acid.

Microglial cells have been implicated as active effectors in neurodegenerative diseases, in which the activated microglia cannot only excessively produce cytotoxic factors, but also initiate anti-inflammatory and immunosuppressive signaling or promote tissue repair and resolution (Ransohoff and Perry, 2009). The unique expression pattern of Siglec-11 on human microglia (Hayakawa et al., 2005) and its ability to interact with SHP-1 and SHP-2 protein tyrosine phosphatases (Angata et al., 2002) have raised great interest in the native function of Siglec-11. Although mice lack the ortholog of Siglec-11, murine microglia culture is a suitable system to study the role of ectopically expressed Siglec-11 since the intracellular signaling pathways between mice and humans are highly conserved. Thus, we used murine microglia expressing human Siglec-11 by transduction with lentiviral vectors. We showed that cross-linking of Siglec-11 by a flag-specific antibody on microglia suppressed LPS-induced gene transcription of the proinflammatory mediators IL-1 $\beta$ and NOS2, and likely TNF- $\alpha$. It is known that IL- $1 \beta$, NO, and TNF- $\alpha$ released from activated microglia are toxic to neurons by actively triggering apoptosis in neurons (Hanisch and Kettenmann, 2007). We further demonstrated that Siglec-11-expressing microglial cells were less toxic to neurons leading to reduced neurite and neuronal cell body loss in microglia-neuron cocultures. Several CD33-related
Siglecs have been shown to inhibit activation pathways induced by immunostimulatory receptors (Avril et al., 2005; Crocker et al., 2007). For example, stimulation of Siglec-5 recruited SHP-1 and SHP-2 and efficiently inhibited FceRI-mediated calcium fluxing (Avril et al., 2005). The cytoplasmic tail of Siglec-11 is very closely related to that of Siglec-5 in respect to sequence similarity and conservation of the protein structure (Angata et al., 2002, 2006; Hayakawa et al., 2005). In our experiments, it is possible that Siglec-11 reduced the microglial production of proinflammatory factors such as IL-1 $\beta$ and NOS2 through immunosuppressive signaling, thereby alleviating microglial neurotoxicity. However, we also showed that Siglec-11-expressing microglia have impaired capacity to phagocytose apoptotic neuronal material. Thus Siglec-11 signaling via ITIM might antagonize the phagocytosis-associated ITAM-Syk signaling pathways (Ziegenfuss et al., 2008). Consequently, higher neurite and neuronal cell body density in the cocultures with Siglec-11-expressing microglia might result either from less phagocytosis or reduced production of microglial proinflammatory mediators. Unfortunately our in vitro system does not allow distinction between neurotoxicity mediated by phagocytosis and soluble mediators. Recently, Siglec-9 (Carlin et al., 2009) and Siglec-10 (Chen et al., 2009) have been shown to dampen innate immune reactivity by recognizing specifically sialylated counterreceptors. Our data illustrated that Siglec-11 is another inhibitory ITIM-bearing CD33-related Siglec that can limit damage by innate immune cells during homeostasis or brain inflammation.

PSA, the long linear homopolymers of Neu5Ac- $\alpha 2-8$, appears to be a ligand of Siglec-11. Expression of PSA is abundant on neurons and glial cells of the developing CNS, but is downregulated in the adult. Very little PSA has been found in nonneural tissues, with the exception of immune cells. PSA was found on the cell surface of natural killer cells (Moebius et al., 2007). PSA was also associated with glycoproteins expressed on dendritic cells (Curreli et al., 2007). Polysialylation of neuropilin-2 on dendritic cells modulated the activation of T cells (Curreli et al., 2007). We observed that PSA is also present on cultured microglia, which might impact their capacity to activate T cells. Removal of PSA from NCAM of the neurons, but not the microglia, had a significant effect on the cytotoxic impact of microglia directed against neurons, indicating that Siglec-11 on microglia was mainly activated by PSA in "trans," but not in "cis."

In summary, we identified a splice variant of Siglec-11 in human microglia, which mediated anti-inflammatory signaling, inhibited phagocytosis and alleviated neurotoxicity of microglia by interaction with PSA on neurons in "trans." Our findings shed new light on the function of the human-specific members of the Siglec family and indicate possible new therapeutic interventions of microglial Siglec-11 in neurodegenerative diseases.

\section{References}

Angata T, Kerr SC, Greaves DR, Varki NM, Crocker PR, Varki A (2002) Cloning and characterization of human Siglec-11. A recently evolved signaling that can interact with SHP-1 and SHP-2 and is expressed by tissue macrophages, including brain microglia. J Biol Chem 277:24466-24474.

Angata T, Hayakawa T, Yamanaka M, Varki A, Nakamura M (2006) Discovery of Siglec-14, a novel sialic acid receptor undergoing concerted evolution with Siglec-5 in primates. FASEB J 20:1964-1973.

Angata T, Tabuchi Y, Nakamura K, Nakamura M (2007) Siglec-15: an immune system Siglec conserved throughout vertebrate evolution. Glycobiology 17:838-846.

Avril T, Freeman SD, Attrill H, Clarke RG, Crocker PR (2005) Siglec-5 (CD170) can mediate inhibitory signaling in the absence of immunore- 
ceptor tyrosine-based inhibitory motif phosphorylation. J Biol Chem 280:19843-19851.

Bally-Cuif L, Goridis C, Santoni MJ (1993) The mouse NCAM gene displays a biphasic expression pattern during neural tube development. Development 117:543-552.

Biber K, Neumann H, Inoue K, Boddeke HW (2007) Neuronal 'On' and 'Off' signals control microglia. Trends Neurosci 30:596-602.

Cao H, Lakner U, de Bono B, Traherne JA, Trowsdale J, Barrow AD (2008) SIGLEC16 encodes a DAP12-associated receptor expressed in macrophages that evolved from its inhibitory counterpart SIGLEC11 and has functional and non-functional alleles in humans. Eur J Immunol 38:2303-2315.

Cao H, de Bono B, Belov K, Wong ES, Trowsdale J, Barrow AD (2009) Comparative genomics indicates the mammalian CD33rSiglec locus evolved by an ancient large-scale inverse duplication and suggests all $\mathrm{Si}-$ glecs share a common ancestral region. Immunogenetics 61:401-417.

Cardona AE, Pioro EP, Sasse ME, Kostenko V, Cardona SM, Dijkstra IM, Huang D, Kidd G, Dombrowski S, Dutta R, Lee JC, Cook DN, Jung S, Lira SA, Littman DR, Ransohoff RM (2006) Control of microglial neurotoxicity by the fractalkine receptor. Nat Neurosci 9:917-924.

Carlin AF, Uchiyama S, Chang YC, Lewis AL, Nizet V, Varki A (2009) Molecular mimicry of host sialylated glycans allows a bacterial pathogen to engage neutrophil Siglec-9 and dampen the innate immune response. Blood 113:3333-3336.

Chen GY, Tang J, Zheng P, Liu Y (2009) CD24 and Siglec-10 selectively repress tissue damage-induced immune responses. Science 323:17221725.

Crocker PR (2005) Siglecs in innate immunity. Curr Opin Pharmacol 5:431-437.

Crocker PR, Paulson JC, Varki A (2007) Siglecs and their roles in the immune system. Nat Rev Immunol 7:255-266.

Curreli S, Arany Z, Gerardy-Schahn R, Mann D, Stamatos NM (2007) Polysialylated neuropilin-2 is expressed on the surface of human dendritic cells and modulates dendritic cell-T lymphocyte interactions. J Biol Chem 282:30346-30356.
Gorlovoy P, LarionovS, Pham TT, Neumann H (2009) Accumulation of tau induced in neurites by microglial proinflammatory mediators. FASEB J 23:2502-2513.

Hanisch UK, Kettenmann H (2007) Microglia: active sensor and versatile effector cells in the normal and pathologic brain. Nat Neurosci 10:13871394.

Hayakawa T, Angata T, Lewis AL, Mikkelsen TS, Varki NM, Varki A (2005) A human-specific gene in microglia. Science 309:1693.

Moebius JM, Widera D, Schmitz J, Kaltschmidt C, Piechaczek C (2007) Impact of polysialylated CD56 on natural killer cell cytotoxicity. BMC Immunol 8:13.

Neumann H, Kotter MR, Franklin RJ (2009) Debris clearance by microglia: an essential link between degeneration and regeneration. Brain 132: 288-295.

Ransohoff RM, Perry VH (2009) Microglial physiology: unique stimuli, specialized responses. Annu Rev Immunol 27:119-145.

Rutishauser U (2008) Polysialic acid in the plasticity of the developing and adult vertebrate nervous system. Nat Rev Neurosci 9:26-35.

Takahashi K, Rochford CD, Neumann H (2005) Clearance of apoptotic neurons without inflammation by microglial triggering receptor expressed on myeloid cells-2. J Exp Med 201:647-657.

Varki A (2009) Natural ligands for CD33-related Siglecs? Glycobiology 19:810-812.

Varki A, Angata T (2006) Siglecs-the major subfamily of I-type lectins. Glycobiology 16:1R-27R.

Zhao J, Brooks DM, Lurie DI (2006) Lipopolysaccharide-activated SHP-1deficient motheaten microglia release increased nitric oxide, TNF-alpha, and IL-1beta. Glia 53:304-312.

Ziegenfuss JS, Biswas R, Avery MA, Hong K, Sheehan AE, Yeung YG, Stanley ER, Freeman MR (2008) Draper-dependent glial phagocytic activity is mediated by Src and Syk family kinase signalling. Nature 453:935-939.

Zujovic V, Benavides J, Vigé X, Carter C, Taupin V (2000) Fractalkine modulates TNF-alpha secretion and neurotoxicity induced by microglial activation. Glia 29:305-315. 